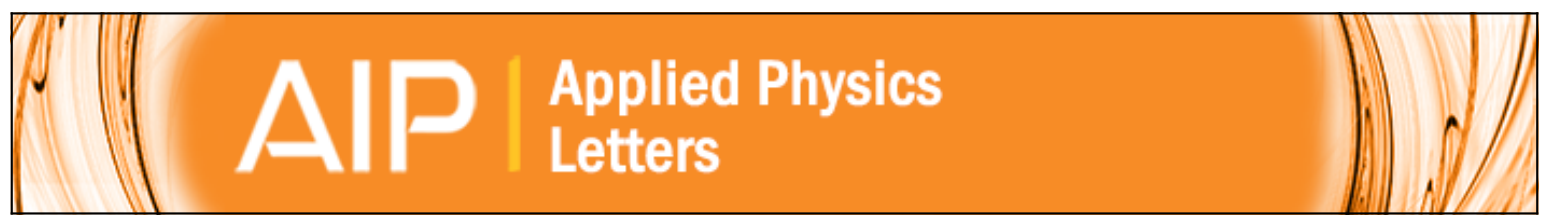

\title{
Comprehensive modeling of resistive switching in the Al / TiO x / TiO 2 / Al heterostructure based on space-charge-limited conduction
}

Sungho Kim, Hu Young Jeong, Sung-Yool Choi, and Yang-Kyu Choi

Citation: Applied Physics Letters 97, 033508 (2010); doi: 10.1063/1.3467461

View online: http://dx.doi.org/10.1063/1.3467461

View Table of Contents: http://scitation.aip.org/content/aip/journal/apl/97/3?ver=pdfcov

Published by the AIP Publishing

\section{Articles you may be interested in}

Space-charge-limited currents and trap characterization in coaxial AIGaN/GaN nanowires

J. Appl. Phys. 110, 044303 (2011); 10.1063/1.3622145

Space-charge limited conduction in doped polypyrrole devices

J. Appl. Phys. 107, 093716 (2010); 10.1063/1.3373393

Field-induced resistive switching based on space-charge-limited current

Appl. Phys. Lett. 90, 022907 (2007); 10.1063/1.2430912

Field-dependent mobility from space-charge-limited current-voltage curves

J. Appl. Phys. 92, 5310 (2002); 10.1063/1.1511825

Space-charge-limited currents in materials with Gaussian energy distributions of localized states Appl. Phys. Lett. 79, 4154 (2001); 10.1063/1.1424046

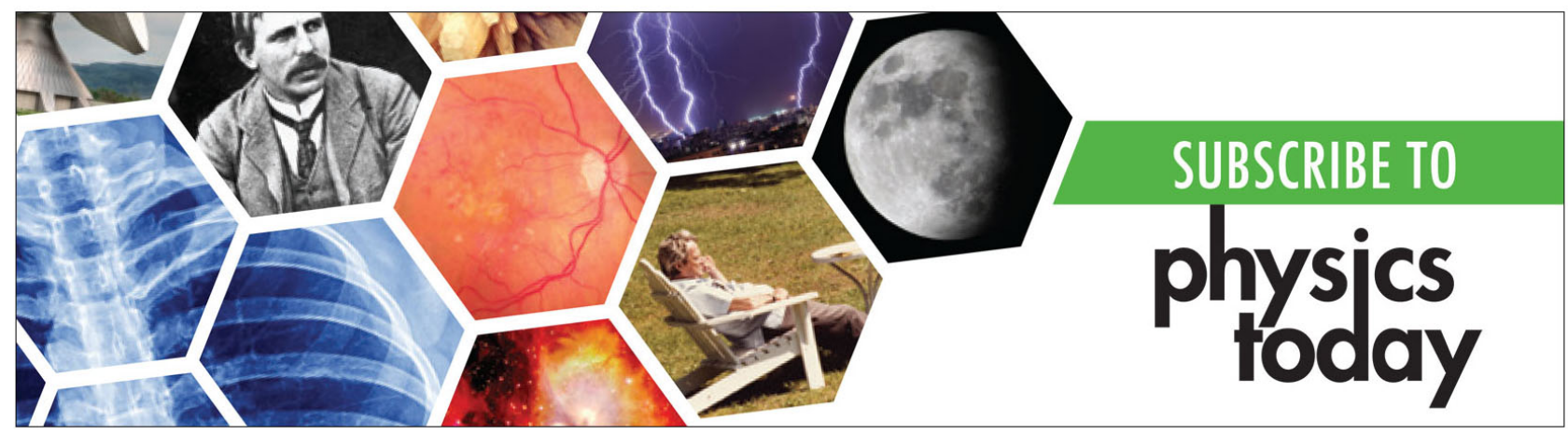




\title{
Comprehensive modeling of resistive switching in the $\mathrm{Al} / \mathrm{TiO}_{\mathrm{x}} / \mathrm{TiO}_{2} / \mathrm{Al}$ heterostructure based on space-charge-limited conduction
}

\author{
Sungho Kim, ${ }^{1} \mathrm{Hu}$ Young Jeong, ${ }^{2}$ Sung-Yool Choi, ${ }^{3}$ and Yang-Kyu Choi ${ }^{1, a)}$ \\ ${ }^{1}$ Department of Electrical Engineering, KAIST, Daejeon 305-701, Republic of Korea \\ ${ }^{2}$ Department of Materials Science and Engineering, KAIST, Daejeon 305-701, Republic of Korea \\ ${ }^{3}$ Electronics and Telecommunications Research Institute (ETRI), Daejeon 305-700, Republic of Korea
}

(Received 26 January 2010; accepted 30 June 2010; published online 23 July 2010)

\begin{abstract}
The reversible resistance switching (RS) effect of the $\mathrm{Al} / \mathrm{TiO}_{\mathrm{x}} / \mathrm{TiO}_{2} / \mathrm{Al}$ heterostructure is investigated in this study. This RS was attributed to space-charge-limited conduction (SCLC) as controlled by localized traps in the $\mathrm{TiO}_{\mathrm{x}}$ layer. The preexisting SCLC theory was extended to describe the abrupt resistance transition. An analytical model was developed with consideration of the ratio of free and trapped carrier density, which was extracted from the experimental data to show exponentially distributed traps in energy. The proposed model can be applicable to RS phenomena induced by interface-type traps in other material system. (c) 2010 American Institute of Physics. [doi:10.1063/1.3467461]
\end{abstract}

Resistance random access memory has recently attracted a great deal of scientific and technological interest related to next-generation memory applications in which resistance can be repeatedly switched by an applied voltage. ${ }^{1}$ This reversible resistance switching (RS) effect has been observed in many types of material systems. A variety of theoretical models were thus proposed to explain the mechanism of RS. One of these models is space-charge-limited conduction (SCLC) theory, which has been employed to understand RS in various materials. ${ }^{2-6}$ Essentially, SCLC is based on the bulk-limited process in which the current flow is governed by the bulk properties of the material. ${ }^{7}$ However, a few papers have reported trap-controlled SCLC behavior originates from localized traps in the metal/oxide interface region. ${ }^{4-6}$ This effect of interface-type traps in SCLC, unfortunately, had not been recognized earlier, although SCLC measurements have been performed for over 50 years. ${ }^{8}$

In a previous work by the authors, the RS behavior of an $\mathrm{Al}$ (top) $/ \mathrm{TiO}_{\mathrm{x}} / \mathrm{TiO}_{2} / \mathrm{Al}$ (bottom) heterostructure was investigated. It was verified that oxygen vacancies are localized in $\mathrm{TiO}_{\mathrm{x}}$ layer and that they act as traps for electrons. ${ }^{9}$ Although trap-controlled SCLC characteristics were clearly observed, a physical analysis could not explain the effect of localized interface-type traps on SCLC at that time. ${ }^{4}$ This letter focuses on the physical analysis of the RS effects in the aforementioned heterostructure and proposes an analytical model to explain the effect of interface-type traps on SCLC.

Figure 1 shows the typical current-voltage $(I-V)$ characteristics of the $\mathrm{Al}$ (top) $/ \mathrm{TiO}_{\mathrm{x}} / \mathrm{TiO}_{2} / \mathrm{Al}$ (bottom) device that produces bipolar resistive switching. Details of the fabrication process of device are available in the literature. ${ }^{9}$ For the electrical measurement, a bias voltage $\left(V_{a}\right)$ was applied to the top electrode while bottom electrode was grounded. When $V_{a}$ was applied in the direction of $0 \mathrm{~V} \rightarrow-3 \mathrm{~V}$ $\rightarrow 0 \mathrm{~V} \rightarrow 3 \mathrm{~V} \rightarrow 0 \mathrm{~V}$, a high-resistance state and a lowresistance state were switched by a specific bias consisting of a negative set voltage $\left(V_{S E T}\right)$ and a positive reset voltage $\left(V_{\text {RESET }}\right)$. The inset of Fig. 1 shows a scan of the atomic profile according to an energy-dispersive $\mathrm{x}$-ray spectrometry

\footnotetext{
a) Author to whom correspondence should be addressed. Electronic mail: ykchoi@ee.kaist.ac.kr.
}

(EDX) analysis. An oxygen-deficient $\mathrm{TiO}_{\mathrm{x}}$ layer was preferentially created at the $\mathrm{Al}$ (top) $/ \mathrm{TiO}_{2}$ interface, creating $\mathrm{Al} / \mathrm{TiO}_{\mathrm{x}} / \mathrm{TiO}_{2} / \mathrm{Al}$ heterostructure naturally. ${ }^{9}$

To investigate the conduction mechanism, doublelogarithmic $I-V$ curves were plotted at the negative voltage sweep region, as shown in Fig. 2. In the negative voltage sweep, the $I-V$ curve shows linear behavior under low voltage and then follows a quadratic form. This charge transport behavior can be explained in terms of the one-carrier (i.e., electron) injected trap-controlled SCLC mechanism. ${ }^{7,10}$ The linear behavior at a low voltage follows Ohm's law due to the thermally generated free carriers inside the film; this is given by $J_{\text {thermal }}=q n_{0} \mu_{\text {eff }} \times\left(V_{a} / d\right)$, where $n_{0}$ is the density of thermally generated free electrons, $\mu_{\text {eff }}$ is the effective electron mobility, and $d$ is the film thickness. To describe the quadratic behavior, SCLC with no traps can be given by $J$ $=(9 / 8) \varepsilon \mu \times\left(V_{a}^{2} / d^{3}\right)$, where $\varepsilon$ is the permittivity of the film. In addition, when the traps are confined in a single discrete energy level, the functional form of trap-controlled SCLC is given by $J=(9 / 8) \varepsilon \mu \theta \times\left(V_{a}^{2} / d^{3}\right)$ in which $\theta$ is described as $\theta=n_{\text {free }} /\left(n_{\text {free }}+n_{\text {trap }}\right)$. Here, $n_{\text {free }}$ is the density of free electrons and $n_{\text {trap }}$ is the density of trapped electrons.

Nevertheless, the total current density $\left(J_{\text {total }}\right)$ is well fitted to SCLC apart from a region of abrupt current transition, as shown in Fig. 2, the effect of interface-type traps localized in the $\mathrm{TiO}_{\mathrm{x}}$ layer should be carefully considered because

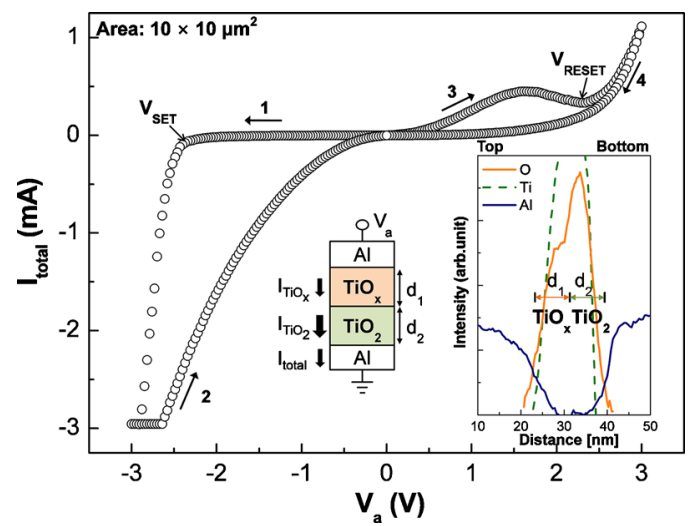

FIG. 1. (Color online) Typical $I-V$ curve of an $\mathrm{Al} / \mathrm{TiO}_{\mathrm{x}} / \mathrm{TiO}_{2} / \mathrm{Al}$ device. The inset shows EDX depth profiling data. 


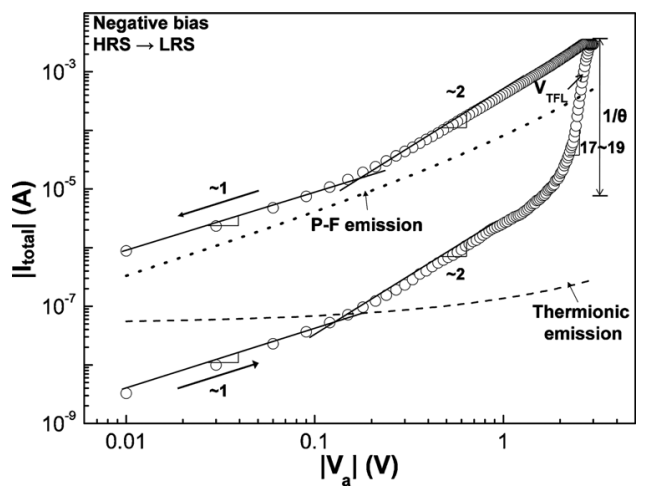

FIG. 2. $I-V$ characteristics in a double-logarithmic plot at negative voltage sweep. Arrows indicate the direction of the voltage sweep. The values of different slopes are listed in the plots. For comparison, Poole-Frenkel emission (dotted line) and thermionic emission (dashed line) are also included.

SCLC is the bulk-limited process. From the inset of Fig. 1, it can be approximated that the thickness of the $\mathrm{TiO}_{\mathrm{x}}$ layer $\left(d_{1}\right)$ is roughly equal to the thickness of the $\mathrm{TiO}_{2}$ layer $\left(d_{2}\right)$. In addition, it should be noted that oxygen vacancies (i.e., traps for electron capturing) are localized only in the $\mathrm{TiO}_{\mathrm{x}}$ layer compared to the $\mathrm{TiO}_{2}$ layer; their distribution can be assumed to be uniform because $d_{1}$ is very thin $(\sim 7 \mathrm{~nm})$. Accordingly, if $\mathrm{TiO}_{2}$ and $\mathrm{TiO}_{\mathrm{x}}$ layers are considered as a trapfree insulator and a trap-including insulator, the current flow of the $\mathrm{TiO}_{2}$ and $\mathrm{TiO}_{\mathrm{x}}$ layers can be described by $J_{\mathrm{TiO}_{2}}$ $=(9 / 8) \varepsilon \mu\left(V_{a}^{2} / d_{2}^{3}\right)$ and $J_{\mathrm{TiO}_{\mathrm{x}}}=(9 / 8) \varepsilon \mu \theta\left(V_{a}^{2} / d_{1}^{3}\right)$, respectively. As $\mathrm{TiO}_{2}$ and $\mathrm{TiO}_{\mathrm{x}}$ layers are serially connected in the heterostructure, the total current flow is determined by the lessconductive part of the entire films. Therefore, $J_{\text {total }}$ is dominantly determined by $J_{\mathrm{TiO}_{\mathrm{x}}}$ because $J_{\mathrm{TiO}_{\mathrm{x}}}$ is always smaller than $J_{\mathrm{TiO}_{2}}(\theta \leq 1)$ when $d_{1} \approx d_{2}$. This approach allows SCLC theory to be applied to the conduction mechanism of an insulating material which has localized interface-type traps. If the thickness of a bulk region is scaled to a comparable thickness of the trap-including interface region, the total conduction can be interpreted by the trap-controlled SCLC theory, despite the fact that SCLC is a bulk-limited process.

In order to model the abrupt RS behavior analytically according to the modulation of $J_{\mathrm{TiO}_{\mathrm{x}}}$, however, the preexisting trap-controlled SCLC theory is not sufficient because analytic solution for $\theta$ is only derived under the condition of $\theta \ll 1$. When abrupt RS occurs, the value of $n_{\text {trap }}$ is saturated to the total trap density $\left(N_{t}\right)$ and injected carriers then start to contribute to the increase in $n_{\text {free }}$. Accordingly, it can be expected that $\theta$ is increased to unity according to $\theta$ $=n_{\text {free }} /\left(n_{\text {free }}+n_{\text {trap }}\right)$. Therefore, analytic expressions of $\theta$ should be derived considering the abrupt RS behavior.

To derive the analytic expressions, $\theta$ can be categorized into three distinctive sections, as shown in Fig. 3(a). First, most traps are empty when $\theta$ is in section A. $\theta$ in section A was previously derived by conventional trap-controlled SCLC theory with the assumption of $\theta \ll 1 ; \theta=\left(N_{c} / N_{t}\right)$ $\times \exp \left[-\left(E_{c}-E_{\text {trap }}\right) / k T\right]$, where traps are located at a single energy level $\left(E_{\text {trap }}\right){ }^{11,12}$ Here, $N_{c}$ is the effective density of states in the conduction band and $E_{c}$ is electron energy at the conduction band minima. Next, in the case of section $C$, all traps are filled by an application of $V_{\mathrm{TFL}}$, which is voltage required to fill the traps. Accordingly, $n_{\text {trap }}$ is equal to $N_{t}$, and injected carriers $\left(n_{\text {injection }}\right)$ are attributed to the increase in $n_{\text {free }}$ At this point it can be deduced that $n_{\text {injection }}=n_{\text {free }}$
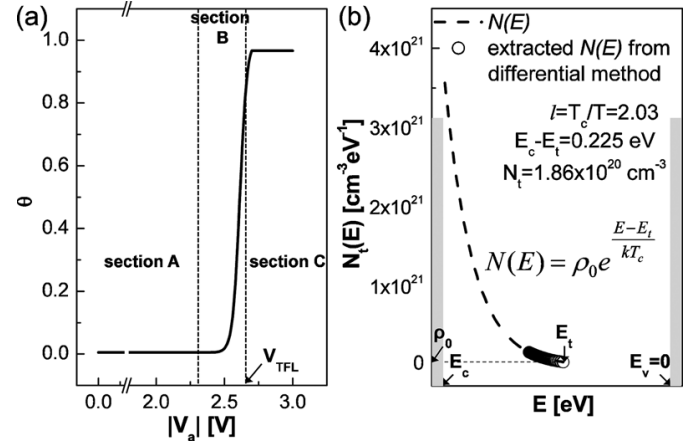

FIG. 3. (a) Schematic of the derivation of the analytic expression of $\theta$. (b) Extracted $N(E)$ using the differential method.

$=N_{c} \exp \left[q\left(V_{a}-V_{\mathrm{TFL}}\right) / k T\right] .{ }^{13}$ Consequently, $\theta$ in section $\mathrm{C}$ is expressed by $\theta=1 /\left\{1+\left(N_{t} / N_{c}\right) \exp \left[-q\left(V_{a}-V_{\mathrm{TFL}}\right) / k T\right]\right\}$. Last, in section $\mathrm{B}$, some of the injected electrons are trapped, and remaining injected electrons contribute to the increase in $n_{\text {free }}$. Consequently, $n_{\text {free }}$ is defined as $n_{\text {injection }}-n_{t, \text { inj }}$, where $n_{t, \text { inj }}$ is the trapped electron density of all of the injected carriers. In addition, $n_{\text {trap }}$ is defined as $n_{t, \text { pre }}$, where $n_{t, \text { pre }}$ is the preexisting trapped electron density. Therefore, $\theta$ is represented by $\theta=n_{\text {free }} /\left(n_{\text {free }}+n_{\text {trap }}\right)=\left(n_{\text {injection }}-n_{t, \text { inj }}\right) /\left(n_{\text {injection }}\right.$ $\left.-n_{t, \text { inj }}+n_{t, \text { pre }}\right)$, which can be approximated to $\theta \approx 1-1 /$ $\left\{1+\left(n_{\text {injection }} / n_{t, \text { pre }}\right)\right\}$ when $n_{t, \text { inj }} \ll n_{t, \text { pre }}$. This approximation is valid when most traps are filled by electrons.

$n_{t, \mathrm{pre}}$ and $n_{t, \mathrm{inj}}$ are determined by the distribution of traps and the related quasi-Fermi level $\left(E_{f n}\right)$ according to $V_{a}{ }^{14}$ The distribution of the trap density as a function of the energy level $E$ and distance $x$ from the top electrode can be written as $H(E, x)=N(E) S(x)$, where $N(E)$ and $S(x)$ represent the energy and spatial distribution functions of the traps, respectively. It was previously assumed that the spatial trap distribution is uniform within the $\mathrm{TiO}_{\mathrm{x}}$ layer; thus, $S(x)=1$. When it is assumed that the traps are confined in a single discrete energy level within the forbidden energy gap, $H(E, x)$ becomes $H(E)=N(E)=N_{t} \delta\left(E-E_{\text {trap }}\right)$. As the trapped electron density follows Fermi-Dirac statistics $\left(f_{n}(E)=1 /\right.$ $\left\{1+\exp \left[\left(E-E_{f n}\right) / k T\right]\right\}$, where $E_{f n}$ is a quasi-Fermi level $),{ }^{7}$ $n_{t, \text { pre }}$ and $n_{t, \text { inj }}$ can be reformulated as $n_{t, \text { pre }}=N_{t} f_{n}\left(E_{\text {trap }}\right)$ and $n_{t, \text { inj }}=\left(N_{t}-n_{t, \text { pre }}\right) f_{n}\left(E_{\text {trap }}\right)$, respectively. As a result, $\theta$ in section $\mathrm{B}$ can be derived when the traps are confined in a single discrete energy level, as $\theta \approx 1-1 /$ $\left\{1+\left(N_{c} / N_{t}\right) \exp \left[q\left(V_{a}-V_{\mathrm{TFL}}\right) / k T\right]\right\}$.

However, it is more feasible to assume that the traps are exponentially distributed in energy than to assume they are located at a single level. Therefore, $N(E)$ becomes $N(E)$ $=\rho_{0} \exp \left[\left(E-E_{t}\right) / k T_{c}\right]$, where $\rho_{0}$ is the trap density at $E=E_{t}$; $N(E)$ becomes the minimum at $E_{t}$ somewhere in the gap and the maximum at $E_{c}$, as shown in Fig. 3(b). $T_{c}$ is a characteristic temperature related to the trap distribution, and $l$ is defined as $l \equiv T_{c} / T{ }^{15,16}$ From the SCLC theory, $N(E)$ can be extracted from the $I-V$ relationship through a variety of methods. Among them, the differential method is employed here to extract $N(E)$, as shown in Fig. 3(b). ${ }^{17}$ Using the $N(E)$ as extracted from the differential method, $N(E), E_{t}$, and $l$ can be determined. Accordingly, $n_{t \text { pre }}$ is determined by evaluating $\int_{E_{t}}^{E_{c}} N(E) f_{n}(E) d E$. Given that an analytical evaluation of this integration is not straightforward, proper simplification is required. At $E_{t}<E<E_{f n}$, the Fermi distribution is close to unity whereas it becomes approximate at $E_{0} \rightarrow E_{\text {fn }}$. It can be 


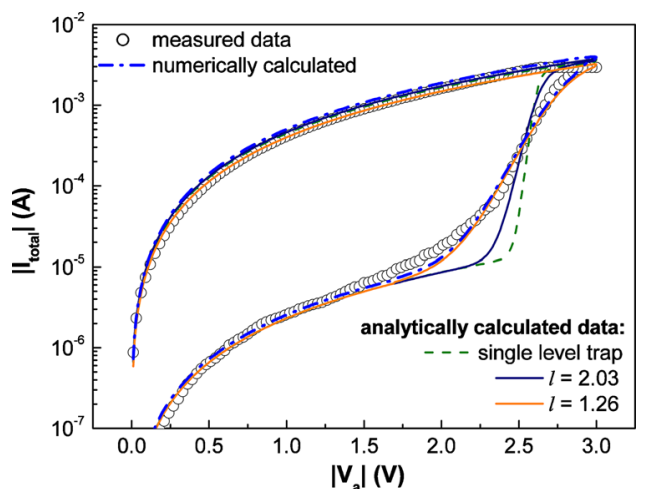

FIG. 4. (Color online) Measured and calculated $I-V$ characteristics at a negative voltage sweep with different types of trap distributions

confirmed by numerical integration that this assumption feasibly gives an approximated solution (as discussed later). Thus, it is possible to obtain $n_{t, \text { pre }} \approx \int_{E_{t}}^{E_{f n}} N(E) d E=N_{t}$ $\times \exp \left[-\left(E_{c}-E_{f n}\right) / k T_{c}\right]$ and $n_{t, \text { inj }} \approx \int_{E_{f n}}^{E_{c}} N(E) f_{n}(E) d E$. With the aid of Boltzmann approximation, this can be simplified to $n_{t, \text { inj }} \approx\left(N_{t} / l-1\right) \exp \left[-\left(E_{c}-E_{f n}\right) / k T_{c}\right]$. Consequently, $\theta$ in section $\mathrm{B}$ can be derived when the traps are distributed exponentially in energy, as $\theta \approx 1-1 /\left\{1+\left(N_{c} / N_{t}\right) \exp \left[q\left(V_{a}-V_{\mathrm{TFL}}\right)\right.\right.$ $\left.\left.\times(l-1) / k T_{c}\right]\right\}$.

Finally, $J_{\text {total }}$ can be determined by the sum of $J_{\text {thermal }}$ for linear behavior at a low voltage and $J_{\mathrm{TiO}_{\mathrm{x}}}$ for quadratic behavior at a high voltage $\left(J_{\text {total }}=J_{\text {thermal }}+J_{\mathrm{TiO}_{\mathrm{x}}}\right) . J_{\mathrm{TiO}_{\mathrm{x}}}$ can be calculated through the use of the derived $\theta$ for the three different sections referred to above. Physical parameters are defined or assumed: 1) $\mu_{\mathrm{eff}}$ is defined as $\mu_{\mathrm{eff}}=\theta \cdot \mu{ }^{7}$ where $\mu$ is $1 \mathrm{~cm}^{2} / \mathrm{V} \mathrm{sec},{ }^{18}$ 2) $N_{c}$ is determined from the effective mass of $\mathrm{TiO}_{2},{ }^{19} m_{\mathrm{TiO}_{2}} \approx 30 m_{0}$; thus, $N_{c}$ is $4.3 \times 10^{21} / \mathrm{cm}^{3}, 3$ ) It is assumed that $n_{0}$ is comparable to thermally injected carriers from the top electrode at zero bias; accordingly, $n_{0}$ $\approx N_{c} \exp \left(-\phi_{b} / k T\right)$, where the Schottky barrier height $\left(\phi_{b}\right)$ at $\mathrm{Al} / \mathrm{TiO}_{\mathrm{x}}$ is $0.1 \sim 0.2 \mathrm{eV}$. Therefore, $n_{0}$ is roughly $10^{15} / \mathrm{cm}^{3}$, and 4) $\varepsilon$ is determined from the relationship $V_{\mathrm{TFL}}$ $=q N_{t} d^{2} / 2 \varepsilon{ }^{7}$. Therefore, $\varepsilon=15.5 \cdot \varepsilon_{0}$, where $\varepsilon_{0}$ is the permittivity of free space. Figure 4 compares the total current calculated from the analytic model and that measured from the fabricated device. When it is assumed that traps are confined in a single discrete energy level, the total current changes very abruptly because all traps are filled when a specific bias (i.e., $V_{\mathrm{TFL}}$ ) is applied (represented by the dotted line in Fig. 4). In contrast, when the traps are distributed exponentially in energy, traps are gradually filled as the bias is increased and the total current gradually changes. When $l=2.03$, as extracted from the differential method in Fig. 3(b), was used to calculate the total current, a small discrepancy was observed between the measured and calculated data. This discrepancy is likely caused by (1) inaccuracy of the differential method and (2) the roughly estimated physical parameters. However, numerically calculated data confirms that the simplified Fermi-Dirac function used for the derivation of $n_{t, \text { pre }}$ and $n_{t, \text { inj }}$ did not lead to the discrepancy. In spite of the discrepancy, the analytically developed $\theta$ suitably describes the RS effect. The best fitted curve was attained when $l=1.26$.

According to the analytical model, it should be expected that the $I-V$ characteristic is symmetric in the positive sweep region. However, Fig. 5 shows that it is asymmetric. The measured double-logarithmic plot and calculated curves at

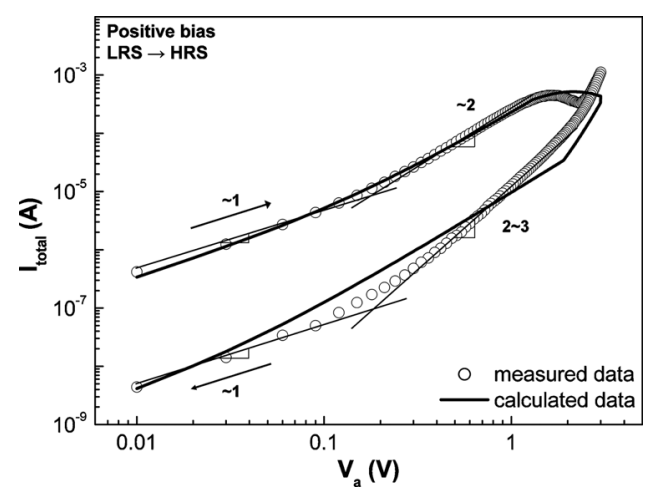

FIG. 5. Measured and calculated $I-V$ characteristics in a double-logarithmic plot at a positive voltage sweep.

the positive voltage sweep region no longer match well, even when $l$ is varied. In another report, a non-symmetric $I-V$ characteristic was understood in terms of the speculation related to the ordering/disordering transition caused by the trap-assist interface phase separation process. ${ }^{6}$ In the present case, it is similarly speculated that some movement of oxygen vacancies which acted as the traps took place when the negative bias was applied. This subsequently changed the trap energy level or the physical properties of the traps. Thus, a different trap-controlled $I-V$ characteristic was observed at a positive voltage sweep. However, details pertaining to this mechanism will be elucidated upon further investigation.

This research was supported by a grant (Grant No. 08K1401-00210) from the Center for Nanoscale Mechatronics \& Manufacturing, one of the 21st Century Frontier Research Programs supported by the Korean Ministry of Education, Science and Technology (MEST).

${ }^{1}$ G. I. Meijer, Science 319, 1625 (2008).

${ }^{2}$ R. Dong, D. S. Lee, W. F. Xiang, S. J. Oh, D. J. Seong, S. H. Heo, H. J. Choi, M. J. Kwon, S. N. Seo, M. B. Pyun, M. Hasan, and H. Hwang, Appl. Phys. Lett. 90, 042107 (2007).

${ }^{3}$ Y. Xia, W. He, L. Chen, X. Meng, and Z. Liu, Appl. Phys. Lett. 90, 022907 (2007).

${ }^{4}$ L.-E. Yu, S. Kim, M.-K. Ryu, S.-Y. Choi, and Y.-K. Choi, IEEE Electron Device Lett. 29, 331 (2008).

${ }^{5}$ N. Baron, Y. Cordier, S. Chenot, P. Vennéguès, O. Tottereau, M. Leroux, F. Semond, and J. Massies, J. Appl. Phys. 105, 033701 (2009).

${ }^{6}$ D. S. Shang, Q. Wang, L. D. Chen, R. Dong, X. M. Li, and W. Q. Zhang, Phys. Rev. B 73, 245427 (2006).

${ }^{7}$ K. C. Kao and W. Hwang, Electrical Transport in Solids (Pergamon, Oxford, New York, 1981).

${ }^{8}$ R. W. I. de Boer and A. F. Morpurgo, Phys. Rev. B 72, 073207 (2005).

${ }^{9}$ S. Kim and Y.-K. Choi, IEEE Trans. Electron Devices 56, 3049 (2009).

${ }^{10}$ Generally, oxygen vacancies in $\mathrm{TiO}_{2}$ are known to act as n-type dopants P. Knauth and H. L. Tuller, J. Appl. Phys. 85, 897 (1999). Therefore, $\mathrm{TiO}_{2}$ is considered as a n-type semiconductor, and its conduction is based on a case of electron-only injected SCLC.

${ }^{11}$ A. Rose, Phys. Rev. 97, 1538 (1955).

${ }^{12}$ M. A. Lampert, Phys. Rev. 103, 1648 (1956).

${ }^{13}$ Actually, $n_{\text {inj }}$ is dependent on the Schottky barrier height $\left(\phi_{b}\right)$ at $\mathrm{Al} / \mathrm{TiO}_{\mathrm{x}}$ hence, $n_{\mathrm{free}}=N_{c} \exp \left[q\left(V_{a}-V_{\mathrm{TFL}}-\phi_{b}\right) / k T\right]$ is more accurate. However, $\phi_{b}$ at $\mathrm{Al} / \mathrm{TiO}_{\mathrm{x}}$ is approximately $0.1-0.2 \mathrm{eV}$. G. Rothenberger, D. Fitzmaurice, and M. Graetzel, J. Phys. Chem. 96, 5983 (1992). Therefore, $\phi_{b}$ is negligible for simplicity of the analytical derivation.

${ }^{14}$ If a quasithermal equilibrium state is assumed when RS is occurred, $E_{f n}$ is obtained by $N_{c} \exp \left[q\left(V_{a}-V_{\mathrm{TFL}}\right) / k T\right]=N_{c} \exp \left[-\left(E_{c}-E_{f n}\right) / k T\right]$.

${ }^{15}$ P. Mark and W. Helfrich, J. Appl. Phys. 33, 205 (1962).

${ }^{16}$ R. S. Muller, Solid-State Electron. 6, 25 (1963).

${ }^{17}$ S. Nešpůrek and J. Sworakowski, J. Appl. Phys. 51, 2098 (1980).

${ }^{18}$ J.-W. Park, D. Lee, H. Kwon, S. Yoo, and J. Huh, IEEE Electron Device Lett. 30, 739 (2009).

${ }^{19}$ T. Bak, J. Nowotny, M. Rekas, and C. C. Sorrell, J. Phys. Chem. Solids 64, 1043 (2003). 